\title{
Lowest Order Mass of KK Graviton Revisited and How It May Affect the Blue Spectrum for Gravitons
}

\author{
Andrew Walcott Beckwith \\ Physics Department, College of Physics, Chongqing University Huxi Campus, Chongqing, China \\ Email: Rwill9955b@gmail.com, abeckwith@uh.edu
}

How to cite this paper: Beckwith, A.W. (2017) Lowest Order Mass of KK Graviton Revisited and How It May Affect the Blue Spectrum for Gravitons. Journal of High Energy Physics, Gravitation and Cosmology, 3, 296-307.

https://doi.org/10.4236/jhepgc.2017.32025

Received: January 19, 2016

Accepted: April 17, 2017

Published: April 20, 2017

Copyright $\odot 2017$ by author and Scientific Research Publishing Inc. This work is licensed under the Creative Commons Attribution International License (CC BY 4.0). http://creativecommons.org/licenses/by/4.0/

\begin{abstract}
The lowest order mass for a KK graviton, as a non-zero product of two branes interacting via a situation similar to Steinhardt's ekpyrotic universe is obtained, as to an alternative to the present dogma specifying that gravitons must be massless. The relative positions as to the branes give a dynamical picture as to how lowest order KK gravitons could be affected by contraction and then subsequent expansion. Initially we have bulk gravitons as a vacuum state. The massless condition is just one solution to a Stern Liuouville operator equation we discuss, which with a non-zero lowest order mass for a KK graviton permits modeling of gravitons via a dynamical Casmir effect which we generalize using Ruser and Duerrer's 2007 work. In particular the blue spectrum for (massless gravitons), is revisited, with consequences for observational astrophysics.
\end{abstract}

\section{Keywords}

Gravitons, Blue Spectrum, KK Theory, Casmir Effect

\section{Introduction}

We make use of work done by Ruser and Duerrer [1] which is essentially a re-do of the Steinhardt model of the ekpyrotic universe, with two branes, one of which is viewed to be stationary and the other is moving toward and away from the stationary brane.

The construction used, largely based upon the Ruser and Duerrer [1] article makes use of a set of differential equations based on the Sturm Liouville method which in the case of the zeroth order mass being zero have in usual parlance a zero value to lowest order KK graviton mass [1]. We will turn this idea on its head by having a non-zero graviton mass, zeroth order in the KK construction as 
to show how graviton mass, lowest order is affected by a Casmir plate treatment of graviton dynamic.

\section{Setting up a Casmir Effect for Zeroth Order "Massive" KK Gravitons}

What we will do is to examine the physics of what is mentioned via figure 1 as in their article as given in [1] the dynamics of the two branes with one stationary and the other moving, which influence a close form solution of the zeroth order graviton mass problem. Figure 1 in this case refers to a figure given in [1] which is not contained in this text.

Using Ruser and Duerrer [1] what we find is that there are two branes on the $A d S_{5}$ space-time so that with one moving and one stationary, we can look at figure 1 as reproduced below which is part of the geometry used in the spatial decomposition of the differential operator acting upon the $h$. Fourier modes of the $h_{i j}$ operator [1]. As given by Ruser and Duerrer, [1] we have that

$$
\left[\partial_{t}^{2}+k^{2}-\partial_{y}^{2}+\frac{3}{y} \cdot \partial_{y}\right] h_{\cdot}=0
$$

Spatially, (1) can be, in its configuration as having

$$
\begin{aligned}
& {\left[\partial_{y}^{2}+\frac{3}{y} \cdot \partial_{y}\right] \Phi_{\alpha}(t, y)} \\
& =m_{\alpha}^{2}(t) \Phi_{\alpha}(t, y)
\end{aligned}
$$

What we will do, instead of looking at a Sturm Liouville operator, as was done in [1] is instead to look at an inner product treatment of the zeroth order mass as can be accessed in a KK decomposition of a graviton, and to consider though using

$$
\left[\partial_{y}^{2}+\frac{3}{y} \cdot \partial_{y}\right] \Phi_{0}(t, y)=m_{0}^{2}(t) \Phi_{0}(t, y)
$$

Standard treatment of the problem represented in (3) is to use the RHS of (3) as set equal to zero. That allows for the "solution" to (3), namely $\Phi_{0}(t, y)=\Phi_{0}=$ constant with respect to space. Our substitution is given below:

An ansatz can be placed into the (3) results above, with, say,

$$
\Phi_{0}(t, y)=\Phi_{0}(\widehat{k}, y)=A \cos (\widehat{k} y)
$$

Our next approximation is to keep the product $k y$ real valued and do a power series expansion of (4) above. Also, we keep the following normalization intact from [1]

$$
\int_{y 1}^{y 2} \frac{\mathrm{d} y}{y^{3}} \cdot \Phi_{\alpha}(y) \Phi_{\beta}(y)=\delta_{\alpha, \beta}
$$

The right hand side is a Kroniker delta, and so it is equal to zero often. So we look at, then if we take an "inner product" procedure as to (4) above we have then the zeroth order mass for a graviton as written up as 


$$
m_{0}^{2}(t)=\widehat{k}^{2} \times\left(1-\left[\frac{-\left.\frac{2}{y^{2}}\right|_{y 1} ^{y 2}-\left.8 \widehat{k}^{2} \ln y\right|_{y 1} ^{y 2}+\left.16 \widehat{k}^{4} y^{2}\right|_{y 1} ^{y 2}-\cdots}{-\left.\frac{2}{y^{2}}\right|_{y 1} ^{y 2}-\left.\widehat{k}^{2} \ln y\right|_{y 1} ^{y 2}+\left.\widehat{k}^{4} y^{2}\right|_{y 1} ^{y 2}-\cdots}\right]\right)
$$

The time dependence as to the above zeroth value comes from looking at if $y 1=y_{b}$, and $y 2=y_{S}$ are such with having, by figure 1 above, $y 1=y_{b}$ moving $y 2=y_{S}$ not able to move, so that (6) definitely has a time dependence. The term $\widehat{k}$ is a term which can be fixed by requirements as to the initial conditions in (5) are met, and equal to 1 when $\alpha=\beta$ and $=0$. The end result is that the (6) is the zeroth order mass term which is not equal to 0.

\section{Lessons from Gryzinski, as Far as Semi-Classical Derivation of a Usually Assumed Quantum Derivation of Inelastic Scattering in Atomic Hydrogen and Its Implications as to (3) and (6)}

We will review the derivation of what is normally assumed to be a quantum result, with the startling implications that a cross section formula, normally quantum, does not need usual Hilbert space construction (usually Hilbert space means quantum mechanics). We will briefly review the Gryzinski result [2] [3] which came from something other than Hilbert space construction and then make our comparison with the likelihood of doing the same thing with respect to forming the zeroth order value of a graviton mass, as not equal to zero, by (3) above without mandating the existence of Hilbert spaces in the electroweak era. Gryzinski [2] [3] starts off with what is called an excitation cross section given by

$$
Q\left(U_{n}\right)=\frac{\sigma_{0}}{U_{n}^{2}} g_{j}\left(\frac{E_{2}}{U_{n}} ; \frac{E_{1}}{U_{n}}\right)
$$

where

$$
g_{j}\left(\frac{E_{2}}{U_{n}} ; \frac{E_{1}}{U_{n}}\right)=\left(\frac{E_{2}}{E_{1}+E_{2}}\right)^{3 / 2} \cdot \Phi
$$

and

$$
\Phi \equiv \frac{2}{3} \cdot \frac{E_{1}}{E_{2}}+\frac{U_{n}}{E_{2}} \cdot\left(1-\frac{E_{1}}{E_{2}}\right)-\left(\frac{U_{n}}{E_{2}}\right) \text { if } U_{n}+E_{1} \leq E_{2}
$$

and

$$
\Phi \equiv\left[\frac{2}{3} \cdot \frac{E_{1}}{E_{2}}+\frac{U_{n}}{E_{2}} \cdot\left(1-\frac{E_{1}}{E_{2}}\right)-\left(\frac{U_{n}}{E_{2}}\right)\right] \cdot \sqrt{ } \text { if } U_{n}+E_{1} \geq E_{2}
$$

with

$$
\sqrt{ }=\sqrt{\left(1+\frac{U_{n}}{E_{1}}\right) \cdot\left(1-\frac{U_{n}}{E_{2}}\right)}
$$

The write up of (7) to (11) has $\sigma_{0}=6.53 \times 10^{-14} \mathrm{~cm}^{2} \mathrm{eV}^{2}$, and $U_{n}$ being 
energy of level n, and $E_{1}$ being the energy of the bound electron, and $E_{2}$ being the energy of the incident electron. We refer the reader to [2] as to what the value of the Born approximation used as a comparison with (11) above. The result was that the Gryzinski's approximation gives scattering cross sections lower than those of the Born approximation although the shape of the curves for cross sectional values are almost the same, with the difference between the Gryzinski approximation and the Born approximation in value closed in magnitude, with principal quantum numbers increased The net effect though is that having a Hilbert space, i.e. assuming that the presence of a Hilbert space implies the Quantum condition, is not always necessary for a typical quantum result. Now, how does that argument as to Hilbert spaces not being necessary for presumed quantum results relate to how to obtain (3)?

\section{In Particular the Blue Spectrum for (Massless Gravitons), Is Revisited, if Gravitons Have a Slight Mass with Consequences for Observational Astrophysics}

We refer to (3) and (6) as giving a non zero value of the zeroth order mass of a graviton in KK theory, and then try to refocus upon the more traditional 4 space definition of $G W$ expansion in order to come up with normal modes. To do this, look at the mode equation in 4 space and its analogy to higher dimensions. In 4 space, the mode equation reads as

$$
\ddot{\chi}_{k}+\left[k^{2}-\frac{\ddot{a}}{a}\right] \chi_{k} \sim \ddot{\chi}_{k}+\left[k^{2}-m_{0}^{2}\right] \chi_{k}=0
$$

Usually $m_{0}=0$, but if it is not equal to zero, then (12) has a more subtle meaning. Consider from Ruser and Duerrer [1] what (12) is turned into, in a more general setting. It gets exotic, namely

$\ddot{q}_{\alpha, k, \boldsymbol{\bullet}}+\left[k^{2}-m_{\alpha}^{2}\right] q_{\alpha, k, \boldsymbol{\bullet}}+\sum_{\beta}\left[M_{\beta, \alpha}-M_{\alpha, \beta}\right] \cdot \dot{q}_{\beta, k, \boldsymbol{\bullet}}+\sum_{\beta}\left[\dot{M}_{\alpha, \beta}-N_{\alpha, \beta}\right] \cdot q_{\beta, k, \boldsymbol{\bullet}}=0$

The obvious connection between the two (12) and (13) is that one will have if $\alpha=0$, then one observes

$$
\sum_{\beta}\left[M_{\beta, \alpha=0}-M_{\alpha=0, \beta}\right] \cdot \dot{q}_{\beta, k, \boldsymbol{\bullet}}+\sum_{\beta}\left[\dot{M}_{\alpha=0, \beta}-N_{\alpha=0, \beta}\right] \cdot q_{\beta, k, \boldsymbol{\bullet}}=0
$$

So, does one have, then, that.

We have, through (13) above outlined an application of Mach's principle as far as the constant value of $\hbar(t)$. Next will be describing how and why Mach's principle can be applied to the gravitino. Note, Mishra [4] used a spin 3/2 particle, and we suggest this is in sync with using a Gravitino.

Mishra, and Mishra \& Christian in [5] came up with a Fermionic particle description of the number of particles in the universe, and since gravitons have spin 2, we are lead to gravitinos of spin 3/2, a super partner description many times larger in mass than the super partner graviton. The Mistra approximation was for a fermionic treatment of kinetic energy as given by $\rho(\boldsymbol{X})$ as a single particle distribution function, such that Mishra used [4] $\rho(\boldsymbol{X}) \equiv A \cdot e^{-x} / x^{3}$, 
where $x=\sqrt{r / \lambda}$, and $r=|\boldsymbol{X}|$, with $\lambda$ a variational parameter, and we have that kinetic energy $K E$ is written as given by [4] [5] and [6]

$$
\langle K E\rangle=\left(\frac{3 \hbar^{2}}{10 m}\right) \cdot\left(3 \pi^{2}\right)^{3 / 2} \cdot \int \mathrm{d} \boldsymbol{X} \cdot[\rho(\boldsymbol{X})]^{5 / 3}
$$

This $\rho(\boldsymbol{X})$ has a normalization such that

$$
\int \mathrm{d} \boldsymbol{X} \cdot[\rho(\boldsymbol{X})]=N
$$

Furthermore, the potential energy is modeled via a Hartree-Fock approximation given by

$$
\langle P E\rangle=-\left(\frac{g^{2}}{2}\right) \cdot \int \mathrm{d} \boldsymbol{X} \cdot \mathrm{d} \boldsymbol{X}^{\prime}\left(\left[\rho(\boldsymbol{X}) \cdot \rho\left(\boldsymbol{X}^{\prime}\right)\right] /\left|\boldsymbol{X}-\boldsymbol{X}^{\prime}\right|\right)
$$

These two were combined together by Mistra to reflect the self-gravitating fictitious particle Hamiltonian [4] [5]

$$
H=-\sum_{i=1}^{N}\left(\frac{\hbar^{2}}{2 m}\right) \cdot \nabla_{i}^{2}-g^{2} \sum_{i=1, i \neq j}^{N} \sum_{j=1}^{N} \frac{1}{\left|\boldsymbol{X}_{i}-\boldsymbol{X}_{j}^{\prime}\right|}
$$

So then a proper spatial averaging of the Hamiltonian will lead, for $\langle H\rangle=E$ quantum energy of the universe given by [4] [5] and [6]

$$
\langle H\rangle=E(\lambda)=\left(\frac{12}{25 \pi}\right) \cdot\left(\frac{\hbar^{2}}{m}\right) \cdot\left(\frac{3 \pi N}{16}\right)^{5 / 3} \cdot \frac{1}{\lambda^{2}}-\left(\frac{g^{2} N^{2}}{16}\right) \cdot \frac{1}{\lambda}
$$

Note that the value $m$, is the mass of the fermionic particle, and that (26) when minimized leads to a minimum energy value of the variational parameter, which at the minimum energy has $\lambda=\lambda_{0}$ for which (26) becomes

$$
E\left(\lambda=\lambda_{0}\right)=E_{0}=-(.015442) N^{7 / 3} \cdot\left(\frac{m g^{4}}{\hbar^{2}}\right)
$$

The tie in with Mach's principle comes as follows; i.e. Mishra sets a net radius value [4] [5] [6]

$$
r=R_{0}=2 \cdot \lambda_{0}=\frac{\hbar^{2}}{m g^{2}} \times(4.0147528) / N^{1 / 3}
$$

This spatial value is picked so that the potential energy of the system becomes equal to the total energy, and note that a total mass, $M$ of the system is computed as follows, i.e. having a mass as given by $M=M_{\text {total }}=N \cdot m$ Mistra, [4] then next assumes that then, there is due to this averaging a tie in, with $M$ being the gravitational mass a linkage to inertial mass so as to write, using (28) and (29) a way to have inertial mass the same as gravitational mass via

$$
E_{g r}=\frac{G \cdot M \cdot m_{\text {grav }}}{R_{0}}=m_{\text {inertial }} \cdot c^{2} \equiv m_{\text {grav }} \cdot c^{2} \Leftrightarrow \frac{G M}{R_{0} c^{2}} \approx 1
$$

This is for total mass $M$ of the universe, and so if we wish to work with a subsystem as what we did with gravitinos, in the electroweak era, we will then change (31) to read instead as a sub set of this Mach's principle, i.e. an electroweak version, i.e. a subset of the Mach's principle 


$$
\frac{G M_{\text {gravitios }}}{R_{E W} c^{2}} \approx \text { const }
$$

We shall outline the consequences of the Machian equation, of the sort given by (32) and from there say something about the limits, next of the Wheeler De Witt equation.

\section{Machian Physics and the Linkage to the Wheeler De Witt Equation and the Limits of the Wheeler De Witt Equation}

Barbour and Pfizer [7] write a very interesting and useful document and interpretation as far as Hamiltonian systems and general relativity. According to [7], the dynamics of general relativity can be written up in terms of a constrained Hamiltonian "with the configuration space for pure gravity being given by the space of all Riemanian metrics on a 3 dimensional manifold $\Sigma$ of fixed but arbitrary topology. We call this topology $Q(\Sigma)$ and have that $g_{a b}(s)$ is the trajectory (of all paths) on $Q(\Sigma)$. In their derivation the vacuum Einstein equations take the form of

$$
g_{a b}^{\prime \prime}+\Gamma_{a b}^{i j k l} g_{i j}^{\prime} g_{k l}^{\prime}=-2 \times\left(R_{a b}-\frac{1}{4} g_{a b} R\right)
$$

This has a Hamiltonian constraint given by

$$
G^{a b c d} g_{a b}^{\prime} g_{c d}^{\prime}-4 \sqrt{g} R=0
$$

And a momentum constraint given by

$$
G^{a b c d} \nabla_{b} g_{c d}^{\prime}=0
$$

Here, $\nabla^{a}$ is the Levi-Civita for a metric $g_{a b}$ with a corresponding Ricci scalar $R$ and Ricci tensor $R^{a b}$ with the $\Gamma_{a b}^{i j k l}$ terms associated with the De Witt metric [20]. As cited by [7], if (25) and (26) are satisfied initially, then by (24), (25) and (26) are continually satisfied Now in what Barbor calls the Machian derivation of General relativity [7] [8] there is one constant linkage of his formalism with the Wheeler De Witt equation, which is that there is no formal time flow, i.e., that the Wheeler De Witt equation in its classical form as in [9] has NO time component added to it. Note that in [8] it is stated that there is no general flow of time, at best there are what Barbor called "time capsules" and that Quantum physics is a way of giving "high probability" to "time capsules". What the author has proposed doing with the Machian perspective is to give a dynamical trajectory as to the Hamiltonian and momentum constraints given as (25) and (26). Needless to say though that what is attempted by (23) is to set up a precondition, independent of (25) and (26) as to set up a configuration for the set of (24), (25) and (26) via (23), and that we regard (24) as a precondition for fulfilling (25) and (26) which are then dynamically satisfied via (24). The idea is that (1) which forms as a byproduct of result of (23) is a precondition for then the formation of the WdW equation as we know it, which we accept as a time independent quantity [9]. 
This construction of the $\mathrm{WdW}$ equation leads to the following question. If Barbor is right about there not being a "flow of time" as we think of it, can we interpret (1) and then (23) as a Machian set up of the WdW equations via (24), (25) and (26)? We submit that what is happening is that if there is no flow of time, that still there is a dynamical set up period, and a conservation of information flow as represented by the formation of $\hbar$ as given in (21) and (22), with then (1), (24) to (26) as preconditions as to keeping the same value of $\hbar$ during cosmological evolution, with the $\mathrm{WdW}$ equation forming after the setup of the initial $\hbar$ which then remains constant.

\section{How to Outline the Resulting Precondition for Constant Value for $\hbar$}

In this note what we do is to organize the interrelationship of the formation of Planck's constant with a necessary and sufficient condition for Quantum processes to form. In a word what we are seeing is that when Planck's constant is being formed, as in the electrodynamic argument given in this paper, that a boundary condition created by Octonian space-time physics exists, which is a boundary of where orthodox QM does not apply and that then later we are applying QM with the formation of Planck's constant after we enter in the regime after the formation of Planck's constant. After the formation of Planck's constant we then are in a position where the Machian relations between gravitinos and gravitons exist, which we claim is a necessary and sufficient condition for a no changing value of $\hbar$. What is done below is to summarize a very sophisticated interrelationship of formation of Planck's constant, the zone of where Octonian geometry no longer holds as separated by a boundary from where Octonian geometry does hold as a necessary and sufficient condition for the onset of using this boundary between Octonionic and non Octonionic geometry as the necessary condition to use relic electromagnetic fields to construct Planck's constant. Note that we are assuming very high electromagnetic fields during and before the electroweak regime [10] which allows, with the presence of a boundary between Octonionic and non Octonionic geometry Planck's constant to form. We summarize our findings as to the results of our discussion in Table 1 as given below.

We have that the formation period for $\hbar$ is our pre quantum regime.

Table 1. Time Interval Dynamical consequences Does QM/WdW apply?

\begin{tabular}{ccc}
\hline Just before & $\begin{array}{c}\text { Form } \hbar \text { from early } E \& M \text { fields, and use Maxwell's } \\
\text { Equations with necessary to implement boundary } \\
\text { Electroweak era } \\
\text { conditions created from change from Octonionic } \\
\text { geometry to flat space }\end{array}$ & $\begin{array}{c}\text { NO } \\
\text { Use (32) as Pre } \\
\text { QM set up }\end{array}$ \\
$\begin{array}{ccc}\hbar \text { kectro-Weak Era constant due to Machian relations } \\
\text { Post }\end{array}$ & $\hbar$ kept constant due to Machian relations & $\begin{array}{c}\text { YES } \\
\text { Use (1) as linkage }\end{array}$ \\
$\begin{array}{c}\text { Electro-Weak Era } \\
\text { to today }\end{array}$ & & $\begin{array}{c}\text { YES } \\
\text { Wave function of } \\
\text { Universe }\end{array}$ \\
\hline
\end{tabular}


This is incidentally the boundary region before the break down of Octonionic gravity, to our present cosmology. When we get to the present era, and the breakdown of Octonionic geometry, exemplified by spatial commutation relations equaling zero, is when QM applies. Before that regime, QM does not apply Furthermore, with the formation of a WdW cosmology, we then have confluence with Barbor's dismissal of the flow of time, as given in [7] and [8] which is in adherence as to [9] in its treatment of the WdW equation as time independent.

\section{Conclusion: Getting the Template as to Keeping Information Content Available for (32) Right}

The Machian hypothesis [7] [10] and actually (9) are a way to address a serious issue. The issue is how to keep the consistency of physical law intact, in cosmological evolution. So far, using the template of gravitons and their superpartners, gravitinos, as information carriers, the author has provided a way to argue that Planck's constant remains invariant as from the EW (electroweak era) to the present era. As one can deduce from physical evolution of the cosmos, time variance of Planck's constant and time variation of the fine structure constant would lead to dramatically different cosmological events than what is deduced by observational astronomy. What we are arguing, using Mach's principle is:

a) Physical law remains invariant in cosmological evolution due to the constant nature/magnitude of $h$ bar, the fine structure constant, and G itself.

b) The linkage in information from a prior to the present universe can be thought of as far as the constancy of (19) concerning gravitinos. While we are aware that gravitinos have a short life time, we argue that (19) would have significant continuity at/before the big bang, and also that this is a way of answering the memory question as to how much cosmological memory is preserved from a prior to the present universe structures. Needless to say though there is a complete breakdown in causality before the formation of the gravitinos which is incidentally the pre-quantum regime of space-time, i.e. where Octonionic geometry predominates.

The main task the author sees is in experimental verification of the following identity. See (27) as below.

The motivation of using two types of Mach's principle, one for the Gravitinos in the electroweak era, and then the 2nd modern day Mach's principle, as organized by the author, is as seen in (27) as re-stated below [10].

$$
\frac{\left.G M_{\text {electro-weak }}\right|_{\text {Super-partner }}}{R_{\text {electro-weak }} c^{2}} \approx \frac{\left.G M_{\text {today }}\right|_{\text {Not-Super-Partner }}}{R_{0} c^{2}}
$$

Once making the double Mach's principle with (27) equal to a constant is done, with $M=N$ times $\mathrm{m}$, where $N$ is the number of a particular particle species, and $m$ is the net mass of the particle species, then an embedding of quantum mechanics using Mach's principle as part of an embedding space can be ventured upon and investigated experimentally. Also, we will be then getting 
ready for the main prize, i.e. finding experimental constraints leading to Planck's constant being invariant. That will do researchers a valuable service as to forming our view of a consistent cosmological evolution of our present cosmology from cycle to cycle. It also would allow for eventually understanding if entropy can also be stated in terms of gravitons alone in early universe models as was proposed by Kiefer \& Starobinsky, et al. [11]. Finally, it would address if QM is embedded in a larger deterministic theory as advocated by t' Hooft [12], the end result would be in examining the following, in terms of $h_{i j}$ values as influenced by massive gravitons. We can use this Machian relationship to understand the $h_{i j}$ values as influenced by massive gravitons. As read from Hinterbichler [13], if $r=\sqrt{x_{i} x_{i}}$, and we look at a mass induced $h_{i j}$ suppression factor put in of $\exp (-m \cdot r)$, then if

$$
\begin{gathered}
h_{00}(x)=\frac{2 M}{3 M_{\text {Planck }}} \cdot \frac{\exp (-m \cdot r)}{4 \pi \cdot r} \\
h_{0 i}(x)=0
\end{gathered}
$$

$h_{i j}(x)$

$=\left[\frac{M}{3 M_{\text {Planck }}} \cdot \frac{\exp (-m \cdot r)}{4 \pi \cdot r}\right] \cdot\left(\frac{1+m \cdot r+m^{2} \cdot r^{2}}{m^{2} \cdot r^{2}} \cdot \delta_{i j}-\left[\frac{3+3 m \cdot r+m^{2} \cdot r^{2}}{m^{2} \cdot r^{4}}\right] \cdot x_{i} \cdot x_{j}\right)$

Here, we have that these $h_{i j}$ values are solutions to the following equation, as given by [13] [14], with $D$ a dimensions value put in.

$$
\left(\partial^{2}-m^{2}\right) h_{\mu v}=-\kappa \cdot\left[T_{u v}-\frac{1}{D-1} \cdot\left(\eta_{u v}-\frac{\partial_{\mu} \partial_{v}}{m^{2}}\right) \cdot T\right]
$$

To understand the import of the above equations, and the influence of the Machian hypothesis, for $G W$ and massive graviton signatures from the electroweak regime, set

$$
\begin{aligned}
& M=10^{50} \times 10^{-27} \mathrm{~g} \equiv 10^{23} \mathrm{~g} \propto 10^{61}-10^{62} \mathrm{eV} \\
& M_{\text {Plank }}=1.22 \times 10^{28} \mathrm{eV}
\end{aligned}
$$

And use the value of the radius of the universe, as given by $r=1.422 \times 10^{27}$ meters, and rather than a super partner gravitino, use the $m_{\text {massive-graviton }} \sim 10^{-26} \mathrm{eV}$ If the $h_{i j}$ values are understood, then we hope we can make sense out of the general uncertainty relationship given by [15]

$$
\left\langle\left(\delta g_{u v}\right)^{2}\left(\hat{T}^{u v}\right)^{2}\right\rangle \geq \frac{\hbar^{2}}{V_{\text {vol }}^{2}}
$$

The hope is to find tests of this generalized uncertainty due to $h_{i j}$ values and to review [13], i.e. to find experimentally falsifiable criteria to determine if Quantum mechanics is actually embedded within a semi classical super structure.

In doing this, we should keep in mind that what Corda brought up in [16] needs to be looked out, i.e. the interferometric tests of general relativity would be an outgrowth of such investigations. 
Furthermore, [17] [18] should be kept in mind in terms of experimental constraints. Gravitational waves have been discovered, and it is opportune for us to keep [17] and [18] in mind when considering the applications of Equation (27) to whatever forms of data sets which may be achievable via experimental gravity.

Last but not least, the author has already had his own version of Equation (33), as seen in [19]. It remains to be seen if [19] is in line with the data sets we may be able to obtain, as well as fidelity with procedures which may allow the issues given in [20] [21] [22] [23] and [24] to be thoroughly looked at from an experimental stand point, as well as [25] for the mass of a graviton.

Finally it would be the gold standard, of determining if initial conditions can be ascertained by data sets to see if [12], as given by t' Hooft holds, i.e. the idea of deterministic conditions for quantum gravity. And possibly the constructions of [26], and [27] as well, provided gravitons having a small pass are not experimentally ruled out.

\section{Acknowledgements}

Thanks to Dr. Corda to suggesting expansion of an initially very incomplete article. The main point of the Gryzinski derivation is that one does not need Hilbert space (usually associated with QM) to obtain what is thought to be a quantum physics result.

This work is supported in part by National Nature Science Foundation of China grant No. 11375279.

\section{References}

[1] Ruser, M. and Durrer, R. Dynamical Casmir Effect in Bouncing Braneworlds. http://theory.physics.unige.ch/ durrer/papers/casilong.pdf

[2] Monnin, C.F. and Prok, G.M. Comparison of Gryzinski's and Born's Approximation for Inelastic Scattering in Atomic Hydrogen. https://ntrs.nasa.gov/archive/nasa/casi.ntrs.nasa.gov/19650017671.pdf

[3] Gryzinski, M. (1959) Classical Theory of Electronic and Ionic Inelastic Collisions. Physical Review, 115, 374-383. https://doi.org/10.1103/PhysRev.115.374

[4] Mishra, S. (2012) The Quantum theory of the Big Bang: Effective theory of Quantum Gravity. In: Connell, J. and Hale, A., Eds., The Big Bang, Theory, Assumptions and Problems, Nova Book Publishers, New York, 205-228.

[5] Mishra, S. and Christian, J. (2011) Effective Theory of Quantum Gravity: Soluble Sector. Abramis Academic Publishing, London.

[6] Christian, J. (1997) Exactly Solvable Sector of Quantum Gravity. Physical Review D, 56, 4844-4877. https://doi.org/10.1103/PhysRevD.56.4844

[7] Barbour, J. and Pfister, H. (1995) Mach's Principle: From Newton's Bucket to Quantum Gravity. Birkhauser, Berlin.

[8] Barbour, J. (2001) The End of Time. Oxford University Press, New York, NY.

[9] Kolb, E. and Turner, S. (1994) The Early Universe. Westview Press, Chicago.

[10] Beckwith, A. (2012) Gravitons, Gravitinos, and Using Mach's Principle to Make a Statement of Equivalent Information in a Gravitino-Graviton Correspondence of Critical Information Exchange from Electroweak to Present Era. 
http://vixra.org/abs/1204.0089

[11] Kiefer, C., Polarski, D. and Starobinsky, A.A. (2000) Entropy of Gravitons Produced in the Early Universe. Physical Review D, 62, Article ID: 043518.

arXiv:gr-qc/9910065

https://doi.org/10.1103/physrevd.62.043518

[12] T’ Hooft, G. (1999) Quantum Mechanics as a Dissipative Deterministic System. http://arxiv.org/PS_cache/gr-qc/pdf/9903/9903084v3.pdf

[13] Hinterbichler, K. (2012) Theoretical Aspects of Massive Gravity. Reviews of Modern Physics, 84, 671-710. http://arxiv.org/abs/1105.3735.pdf https://doi.org/10.1103/RevModPhys.84.671

[14] http://web.mit.edu/redingtn/www/netadv/Xgravitati.html

[15] Downes, T.G. and Milburn, G.J. (2011) Optimal Quantum Estimation for Gravitation. http://xxx.lanl.gov/abs/1108.5220

[16] Corda, C. (2009) Interferometric Detection of Gravitational Waves: The Definitive Test for General Relativity. International Journal of Modern Physics D, 18, 22752282. https://arxiv.org/abs/0905.2502 https://doi.org/10.1142/S0218271809015904

[17] Abbott, B.P., et al. (2016) Observation of Gravitational Waves from a Binary Black Hole Merger. Physical Review Letters, 116, Article ID: 061102. https://doi.org/10.1103/physrevlett.116.061102

[18] Abbott, B.P., et al. (2016) GW151226: Observation of Gravitational Waves from a 22-Solar-Mass Binary Black Hole Coalescence. Physical Review Letters, 116, Article ID: 241103. https://doi.org/10.1103/physrevlett.116.241103

[19] Beckwith, A. (2016) Gedanken Experiment for Refining the Unruh Metric Tensor Uncertainty Principle via Schwarzschild Geometry and Planckian Space-Time with Initial Nonzero Entropy and Applying the Riemannian-Penrose Inequality and Initial Kinetic Energy for a Lower Bound to Graviton Mass (Massive Gravity). Journal of High Energy Physics, Gravitation and Cosmology, 2, 106-124. https://doi.org/10.4236/jhepgc.2016.21012

[20] Avessian, A.K. (2009) Planck's Constant Evolution as a Cosmological Evolution Test for the Early Universe. Gravitation and Cosmology, 15, 10-12. https://doi.org/10.1134/S0202289309010034

[21] Hogan, C. (2002) Holographic Discreteness of Inflationary Perturbations. arXiv:astro-ph/0201020v2

[22] Crowell, L. (2005) Quantum Fluctuations of Space Time. World Scientific Series in Contemporary Chemical Physics, Vol. 25, World Scientific, Singapore.

[23] Beckwith, A. (2011) Octonionic Gravity Formation, Its Connections to Micro Physics. Open Journal of Microphysics, 1, 13-18. https://doi.org/10.4236/ojm.2011.11002

[24] Beckwith, A. (2011) How to Use the Cosmological Schwinger Principle for Energy, Entropy, and "Atoms of Space-Time" to Create a Thermodynamic Space-Time and Multiverse. Journal of Physics. Conference Series, 306, Article ID: 012064. http://iopscience.iop.org/1742-6596/306/1/012064 https://doi.org/10.1088/1742-6596/306/1/012064

[25] Goldhaber, A. and Nieto, M. (2010) Photon and Graviton Mass Limits. Reviews of Modern Physics, 82, 939-979. https://doi.org/10.1103/RevModPhys.82.939

[26] Beckwith, A. (2011) Identifying a Kaluza Klein Treatment of a Graviton Permitting a Deceleration Parameter $\mathrm{Q}(\mathrm{Z})$ as an Alternative to Standard DE. Journal of Cos- 
mology, 13, 1-15. http://journalofcosmology.com/BeckwithGraviton.pdf

[27] Ng, Y.J. (2008) Spacetime Foam: From Entropy and Holography to Infinite Statistics and Nonlocality. Entropy, 10, 441-461. https://doi.org/10.3390/e10040441

Submit or recommend next manuscript to SCIRP and we will provide best service for you:

Accepting pre-submission inquiries through Email, Facebook, LinkedIn, Twitter, etc. A wide selection of journals (inclusive of 9 subjects, more than 200 journals) Providing 24-hour high-quality service User-friendly online submission system Fair and swift peer-review system Efficient typesetting and proofreading procedure Display of the result of downloads and visits, as well as the number of cited articles Maximum dissemination of your research work

Submit your manuscript at: http://papersubmission.scirp.org/ Or contact jhepgc@scirp.org 